
February 1946

\title{
HEAT CAPACITY OF A TWO-PHASE SYSTEM, WITH APPLI- CATIONS TO VAPOR CORRECTIONS IN CALORIMETRY
}

\author{
By Harold J. Hoge
}

\section{ABSTRACT}

A formula is derived that gives the heat capacity of a system composed of solid or liquid in equilibrium with saturated vapor in terms of the specific heat of the condensed phase and certain auxiliary data. This formula is valid throughout the entire range from 0 to 100 percent of vapor, and at the latter extreme reduces to a well-known relation between the specific heats of saturated liquid and saturated vapor.

The formula is applied to the calculation of vapor corrections in calorimetry. Its advantage lies in the fact that the correction is expressed as a single term that may be readily transformed with Clapeyron's equation, yielding two alternative correction formulas.

Vapor corrections to the heat capacity and to the heat of fusion are summarized and tabulated for four different experimental procedures in calorimetry.

\section{CONTENTS}

II. Heat capacity of a two-phase system

III. Vapor correction to the heat of fusion

IV. Application to calorimetry

V. Summary of formulas and required data $\ldots \ldots \ldots \ldots$

VI. Definitions and symbols_._.

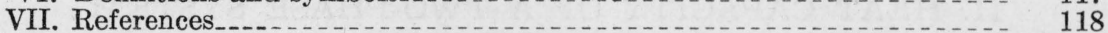

\section{INTRODUCTION}

A solid or liquid having an appreciable vapor pressure will partially vaporize when placed in a closed calorimeter. The presence of vapor, which changes both in volume and density as the temperature is changed, makes it necessary in such cases to apply a vapor correction to heat-capacity data. The size of the correction varies with the degree of filling of the calorimeter. In practice it seldom exceeds 3 or 4 percent of the heat capacity of the solid or liquid, but under unusual conditions has been reported to be as large as 20 percent [1].*

Osborne and Van Dusen [2] appear to have been the first to give a detailed mathematical treatment of the problem of vapor corrections. Their equations are given in the Handbuch der Experimentalphysik [3] and seem to have been widely adopted. In 1924 Osborne [4, 5] gave a new treatment of the problem of vapor corrections based on the practice of making duplicate calorimetric runs, one with a large and the other with a small amount of material in the calorimeter. $\mathrm{He}$ appears to have been the first to use this method, which has

\footnotetext{
* Figures in brackets indicate the literature references at the end of this paper.
} 
several advantages, among which are the elimination both of the effect of material in the filling tube and the effect of strain on the heat capacity of the calorimeter. Others who have discussed vapor corrections, either incidentally or in detail include Babcock [6], Awbery and Griffiths [7], and Bennewitz and Splittgerber [8].

All the correction formulas given by these authors are mathematically and physically correct, but some are unnecessarily complicated. Nowhere has it been clearly brought out that the correction can always be expressed in two alternative forms, one of which is in terms of latent heats, the other in terms of vapor pressures. The present paper is an attempt to clarify the situation, and to present in their simplest form the equations needed for making the vapor correction in any ordinary calorimetric experiment. It is thought that these correction formulas may help to eliminate the unnecessary work that is sometimes done when vapor pressure data are first used to compute latent heats, the latent heats then being used in a vapor correction formula. In such a calculation the specific volume of the vapor appears twice, once in Clapeyron's equation, and again in computing $x$, the fraction of the material in the vapor phase. If incorrect vapor volumes are used, incorrect latent heats are obtained, but this error is exactly compensated by the error in $\mathrm{x}$, so that the vapor correction is independent of the assumed vapor volume. It seems unlikely that investigators have been generally aware of this fact.

The starting point in the derivation given below is an equation for the entropy of a two-phase system. This considerably shortens the derivation as compared with the conventional approach of accounting for all the energy supplied, and yields the results directly in the desired form without the necessity of combining terms. The suggestion that the problem be treated from the entropy standpoint was made by Harold W. Woolley, and it was this point of view that led to equation 5 .

\section{HEAT CAPACITY OF A TWO-PHASE SYSTEM}

Consider a mass $M^{1}$ of material confined in a volume, $V$, and partially vaporized, so that there are $M_{\mathrm{g}}$ grams of gas in equilibrium with $M_{\mathrm{c}}$ grams of condensed phase, which may be either solid or liquid. This system has a definite heat capacity, provided the volume, $V$, is a definite function of temperature, for if this is the case, the state of the system is completely defined by the temperature. The pressure in the system is always the vapor pressure of the material. We wish to find an expression for the heat capacity of this system. This is most easily done by finding an expression for the total entropy of the system, and finally converting this expression by differentiation into an equation giving the desired heat capacity.

Let $s_{\mathrm{g}}$ and $s_{\mathrm{c}}$ be the entropies per unit mass of saturated vapor and saturated condensed phase, respectively. Then the total entropy of the system is

$$
\begin{aligned}
S & =M_{\mathrm{c}} s_{\mathrm{c}}+M_{\mathrm{g}} s_{\mathrm{g}} \\
& =M s_{\mathrm{c}}+M_{\mathrm{g}}\left(s_{\mathrm{g}}-s_{\mathrm{c}}\right) .
\end{aligned}
$$

${ }_{1}$ Symbols are defined as they appear, and 8 table of symbols used is given at the end of this paper. 
Since $s_{\mathrm{g}}-s_{\mathrm{c}}$ is the entropy of vaporization per unit mass it is equal to $l / T$, where $l$ is the heat of vaporization per unit mass, so that

$$
S=M s_{\mathrm{c}}+M_{\mathrm{g}} l / T .
$$

The quantity $M_{\mathrm{g}} l / T$ may be considered an "excess" entropy, since it represents the difference between the entropy of the actual system and that of the corresponding system with no vapor phase. Putting $S^{\prime}=M_{\mathrm{g}} l / T$ we have

$$
S=M s_{\mathrm{c}}+S^{\prime}
$$

and differentiating with respect to $T$

$$
\frac{d S}{d T}=M \frac{d s_{\mathrm{e}}}{d T}+\frac{d S^{\prime}}{d T} .
$$

Now for any reversible absorption of heat $d S=\delta Q / T=C d T / T$. Hence we may substitute $d S / d T=C / T$ and $d s_{\mathrm{e}} / d T=c_{\mathrm{e}} / T$, where $C$ is the heat capacity of the two-phase system and $c_{\mathrm{c}}$ is the heat capacity of unit mass of saturated condensed phase. This gives

$$
C=M c_{\mathrm{c}}+T \frac{d S^{\prime}}{d T},
$$

which is the basic formula for the heat capacity of a two-phase system.

The next problem is to evaluate $S^{\prime} \equiv M_{\mathrm{g}} l / T$. The mass of vapor, $M_{\mathrm{g}}$, can be expressed in terms of the total mass, $M$, the total volume, $V$, and the specific volumes of vapor and condensed phase, $v_{\mathrm{g}}$ and $v_{\mathrm{c}}$, as follows:

whence

$$
\begin{aligned}
V & =M_{\mathrm{c}} v_{\mathrm{c}}+M_{\mathrm{g}} v_{\mathrm{g}} \\
& =M v_{\mathrm{c}}+M_{\mathrm{g}}\left(v_{\mathrm{g}}-v_{\mathrm{c}}\right),
\end{aligned}
$$

$$
M_{\mathrm{q}}=\frac{V-M v_{\mathrm{o}}}{v_{\mathrm{g}}-v_{\mathrm{o}}}
$$

The excess entropy is therefore

$$
S^{\prime} \equiv \frac{M_{\mathrm{g}} l}{T}=\frac{l}{T} \frac{V-M v_{\mathrm{c}}}{v_{\mathrm{g}}-v_{\mathrm{c}}} .
$$

The quantity $l /\left[T\left(v_{\mathbf{g}}-v_{\mathrm{c}}\right)\right]$ is by Clapeyron's equation equal to $d p / d T$, the slope of the vapor-pressure curve. This substitution gives an alternative expression for excess entropy. ${ }^{2}$

$$
S^{\prime}=\frac{d p}{d T}\left(V-M v_{\mathrm{c}}\right) .
$$

Equations $5,7 \mathrm{a}$, and $7 \mathrm{~b}$, permit $c_{\mathrm{c}}$, the specific heat of saturated condensed phase to be calculated from $C$, the heat capacity of the two-phase system, and certain auxiliary data, which may be either $l$, $V, v_{\mathrm{g}}$, and $v_{\mathrm{e}} ;$ or $d p / d T, V$, and $v_{\mathrm{c}}$. When these formulas are used to make vapor corrections they will ordinarily be applied to systems whose vapor fraction, or dryness $\left(=M_{\mathbf{g}} / M\right)$ is between 0 and 10 per-

\footnotetext{
${ }^{2} \mathrm{~A}$ shorter but less obvious method of getting equations $7 \mathrm{a}$ and $7 \mathrm{~b}$ is to note that $S^{\prime}$ is the change in entropy when the volume occupied by the material is increased from $M v$ e to $V$ at constant temperature, so that $S^{\prime}=(\partial S / \partial V)_{T}\left(V-M v_{e}\right)$. Since, by Maxwell's relation $(\partial S / \partial V)_{T}=d p / d T$, we have $7 \mathrm{~b}$ at once and $7 \mathrm{a}$ may be obtained from $7 \mathrm{~b}$ by use of Clapeyron's equation.
} 


\section{Journal of Research of the National Bureau of Standards}

cent. They are valid, however, throughout the whole range from 0 to 100 percent dryness. For the limiting case of 100 percent dryness, 5 and $7 \mathrm{a}$ yield a well-known relation between the heat capacities of saturated vapor and saturated liquid. For this particular case, $V=M v_{\mathrm{g}}$, so that $S^{\prime}$ is simply $M l / T$, and 5 is

$$
C=M c_{\mathrm{g}}=M c_{\mathrm{c}}+T \frac{d}{d T}\left(\frac{M l}{T}\right),
$$

where $c_{\mathrm{g}}$ is the heat capacity per gram of saturated vapor. Since $M$ is independent of $T$, this reduces to the usual form

$$
c_{\mathrm{g}}-c_{\mathrm{c}}=T \frac{d}{d T}\left(\frac{l}{T}\right) .
$$

\section{VAPOR CORRECTION TO THE HEAT OF FUSION}

When a melting point or other first-order transition is encountered in a two-phase system, special formulas are needed for making the vapor correction. The simplest way to obtain these is to start with equation 3 , the equation for the entropy of the two-phase system, $S=M s_{\mathrm{c}}+S^{\prime}$. Let the subscript "a" refer to the system just before the transition starts (solid-gas) and let the subscript "b" characterize it at the end of the transition (liquid-gas). The change in entropy during melting is, by equation 3 ,

$$
S_{\mathrm{b}}-S_{\mathrm{a}}=M\left(s_{\mathrm{cb}}-s_{\mathrm{ca}}\right)+S_{\mathrm{b}}^{\prime}-S_{a}^{\prime} .
$$

If $Q$ is the measured quantity of heat supplied during the process, then $Q / T=S_{\mathrm{b}}-S_{\mathrm{a}}$. Likewise, $s_{\mathrm{cb}}-s_{\mathrm{ca}}$, the difference in specific entropies of the two condensed phases, is $l_{\mathrm{f}} / T$, where $l_{\mathrm{f}}$ is the heat of fusion per gram. Making these substitutions and multiplying by $T$ gives

$$
Q=M l_{\mathrm{f}}+T\left(S_{\mathrm{b}}^{\prime}-S_{a}^{\prime}\right) .
$$

The excess entropies may be evaluated with the formulas previously developed. If $7 \mathrm{a}$ is used,

while if $7 \mathrm{~b}$ is used,

$$
S_{\mathrm{b}}^{\prime}-S_{\mathrm{a}}^{\prime}=\left[\frac{l V-M v_{\mathrm{c}}}{T v_{\mathrm{g}}-v_{\mathrm{c}}}\right]_{\mathrm{a}}^{\mathrm{b}},
$$

$$
S_{\mathrm{b}}^{\prime}-S_{\mathrm{a}}^{\prime}=\left[\frac{d p}{d T}\left(V-M v_{\mathrm{c}}\right)\right]_{\mathrm{a}}^{\mathrm{b}}
$$

When evaluating these expressions it should be remembered that the only quantities having the same value at both limits are $V, M, T$, and $v_{\mathrm{g}}$.

The vapor correction to the heat of fusion may be considered to be made up of two parts. One part is due to the fact that $M_{\mathrm{g}}$ is not zero, and hence not all of the total mass $M$ takes part in the process of melting. The other is due to the fact that $M_{\mathrm{g}}$ generally changes a little one way or the other owing to a difference between $v_{\mathrm{ca}}$ and $v_{\mathrm{cb}}$. This causes the absorption or liberation of the heat of vaporization of a small amount of material. 


\section{APPLICATION TO CALORIMETRY}

Use of the formulas derived above in making vapor corrections is a straightforward procedure that need not be discussed in detail. The vapor correction is not made until after the calculation of the gross, tare, and net heat capacities. In calculating both the gross and the tare heat capacities it may be necessary to apply a curvature correction [reference 9, p. 79]. After this correction has been applied it is convenient to tabulate the gross and tare heat capacities at 5-degree intervals and to subtract one table from the other to obtain the net heat capacity. The vapor correction is then applied to the net heat capacity.

The working formulas for this purpose depend on the type of experiment. Four different types of experiment may be considered, characterized by the presence or absence of a filling tube that contains a small part of the calorimetric charge, and by whether the tare charge is zero or a mass of material $M_{2}$, which is small compared to the gross charge $M_{1}$ but sufficient to fill the system with saturated vapor at all temperatures.

In the next section the appropriate formulas for each of the four types of experiment are tabulated. The quantities which must be known in order to evaluate the formulas are also listed. These are divided into two groups: the basic data, which are required regardless of the vapor correction; and the auxiliary data, which are needed only to evaluate the vapor correction. For one type of experiment, formulas are given which contain $M_{\mathrm{t}}$, the mass" of material in the filling tube. These formulas may be derived by treating the vapor in the tube as an ideal gas. The problem is essentially the same as that of correcting for the gas in the capillary of a gas thermometer [10]. In order that the filling tube shall contain only vapor, it is of course necessary that no part of it shall have a temperature lower than that of the calorimeter proper. ${ }^{3}$

In calculating the vapor correction, the derivative $d E / d T$ must always be calculated, and in many cases the derivative $d p / d T$ also. A procedure which has been found satisfactory for this purpose is to tabulate $E$ (or $p$ ) at 5 -degree intervals and to calculate $\Delta E / \Delta T$ for each interval. The values of $\Delta E / \Delta T$ are ordinarily sufficiently good approximations to the values of $d E / d T$ at the midpoints of the intervals. Where greater accuracy is desired, formulas such as that of Rutledge [11], which are based on polynomial approximations of higher degree may be used.

The author acknowledges valuable discussions with M. S. Van Dusen, of the Bureau's Pyrometry Section.

${ }^{8}$ Osborne and his coworkers have for certain experiments used a filling tube that is at all times filled with liquid. This requires the opposite condition-that no part of the tube shall have a temperature higher than that of the calorimeter proper. 


\section{SUMMARY OF FORMULAS AND REQUIRED DATA}

For definitions of terms and symbols, see section VI.

Type 1. Gross charge: $M$

Tare charge: 0

Filling tube: No

$$
\begin{aligned}
& \left\{\begin{array}{l}
c_{\mathrm{c}}=\frac{C_{\mathrm{net}}-T d S^{\prime} / d T}{M} \\
l_{\mathrm{f}}=\frac{Q-T\left(S_{\mathrm{b}}^{\prime}-S_{\mathrm{a}}^{\prime}\right)}{M} \\
\text { Basic data: } C_{\mathrm{net}}, M, T, Q
\end{array}\right. \\
& \left\{\begin{array}{l}
S^{\prime}=\frac{l}{T} \frac{V-M v_{\mathrm{c}}}{v_{\mathrm{g}}-v_{\mathrm{c}}} \\
\text { Auxiliary data: } l_{\mathrm{a}}, l_{\mathrm{b}}, V, v_{\mathrm{ca}}, v_{\mathrm{cb}}, v_{\mathrm{g}}
\end{array}\right.
\end{aligned}
$$

or

$$
\left\{\begin{array}{l}
S^{\prime}=\frac{d p}{d T}\left(V-M v_{\mathbf{c}}\right) \\
\text { Auxiliary data: }(d p / d T)_{\mathbf{a}},(d p / d T)_{\mathbf{b}}, V, v_{\mathrm{ca}}, v_{\mathrm{cb}} .
\end{array}\right.
$$

Type 2. Gross charge: $M_{1}$

Tare charge: $M_{2}$

Filling tube: No

$$
\left\{\begin{array}{l}
c_{\mathrm{c}}=\frac{C_{\mathrm{net}}-T d\left(S_{1}^{\prime}-S_{2}^{\prime}\right) / d T}{M_{1}-M_{2}} \\
l_{\mathrm{t}}=Q_{\mathrm{net}}-T \frac{\left[\left(S_{1}^{\prime}-S_{2}^{\prime}\right)_{\mathrm{b}}-\left(S_{1}^{\prime}-S_{2}^{\prime}\right)_{\mathrm{a}}\right]}{M_{1}-M_{2}} \\
\text { Basic data: } C_{\text {net }}, M_{1}-M_{2}, T, Q_{\mathrm{net}},
\end{array}\right.
$$

or

$$
\left\{\begin{array}{l}
S_{1}^{\prime}-S_{2}^{\prime}=-T \frac{\left(M_{1}-M_{2}\right) v_{\mathrm{c}}}{v_{\mathrm{g}}-v_{\mathrm{c}}} \\
\text { Auxiliary data: } l_{\mathrm{a}}, l_{\mathrm{b}}, v_{\mathrm{ca}}, v_{\mathrm{cb}}, v_{\mathrm{g}} .
\end{array}\right.
$$

$$
\left\{\begin{array}{l}
S_{1}^{\prime}-S_{2}^{\prime}=-\frac{d p}{d T}\left(M_{1}-M_{2}\right) v_{\mathrm{e}} \\
\text { Auxiliary data: }(d p / d T)_{\mathrm{a}},(d p / d T)_{\mathrm{b}}, v_{\mathrm{ca}}, v_{\mathrm{cb}} .
\end{array}\right.
$$

Type 3. Gross charge: $M_{1}$

Tare charge: $M_{2}$

Filling tube: Yes

The temperature distribution in the tube must be the same in the tare run as in the gross run. With this precaution, the quantities $M_{\mathrm{t}}$ and $d M_{\mathrm{t}} / d T$ drop out of the working equations, which become identical with those for an experiment of type 2 . 
Type 4. Gross charge: $M$

Tare charge: 0

Filling tube: Yes

$$
\begin{aligned}
& \left\{\begin{array}{l}
c_{\mathrm{a}}=\frac{C_{\mathrm{net}}-T d S^{\prime} / d T-l d M_{\mathrm{t}} / d T}{M-M_{\mathrm{t}}} \\
l_{\mathrm{t}}=\frac{Q-T\left(S_{\mathrm{b}}^{\prime}-S_{\mathrm{a}}^{\prime}\right)}{M-M_{\mathrm{t}}} \\
\text { Basic data: } C_{\mathrm{net}}, M, T, Q .
\end{array}\right. \\
& \left\{\begin{array}{l}
S^{\prime}=\frac{l}{T} \frac{V-\left(M-M_{\mathrm{t}}\right) v_{\mathrm{c}}}{v_{\mathrm{g}}-v_{\mathrm{c}}} \\
\text { Auxiliary data: } l_{\mathrm{a}}, l_{\mathrm{b}}, \mathrm{V}, v_{\mathrm{ca}}, v_{\mathrm{cb}} \cdot v_{\mathrm{g}}
\end{array}\right.
\end{aligned}
$$

or

also

$$
\left\{\begin{array}{l}
S^{\prime}=\frac{d p}{d T}\left[V-\left(M-M_{\mathrm{t}}\right) v_{\mathrm{o}}\right] \\
\text { Auxiliary data: }(d p / d T)_{\mathrm{a}},(d p / d T)_{\mathrm{b}}, V, v_{\mathrm{ca}}, v_{\mathrm{eb}}
\end{array}\right.
$$

$$
\left\{\begin{array}{l}
M_{\mathrm{t}}=p_{R}^{W} \sum \frac{V_{\mathrm{t}}}{T_{1}} \\
\frac{d M_{\mathrm{t}}}{d T}=\frac{W}{R}\left[\frac{d p}{d T} \sum_{1} T_{1}+p \frac{d}{d T} \sum_{1} \frac{V_{1}}{T_{1}}\right] \\
\text { Auxiliary data: } p, V_{1}, T_{1}, d p / d T .
\end{array}\right.
$$

\section{DEFINITIONS AND SYMBOLS}

Calorimeter.-That part of the apparatus which contains the material and participates in absorbing the measured power input.

Charge.-Mass of material in the calorimeter and filling tube.

Filling tube.-Tube extending from the calorimeter to outside the apparatus. Contains a small part of the total charge during measurements.

Gross heat capacity.-Heat capacity of calorimeter plus a large charge $M_{1}$.

Tare heat capacity.-Heat capacity of calorimeter with no charge, or with a small charge $M_{2}$.

Net heat capacity.-Gross heat capacity minus tare heat capacity.

$C=$ heat capacity

$c=$ specific heat (heat capacity per unit mass)

$l=$ heat of sublimation, vaporization or fusion per unit mass

$M=$ mass of charge contained in calorimeter and filling tube

$p=$ pressure, equal to vapor pressure of material at temperature $T$

$Q=$ heat absorbed during a transition such as melting

$R=$ gas constant

$S=$ entropy

$S^{\prime}=$ excess entropy of a system, as compared with the same mass of saturated condensed phase

$s=$ entropy per unit mass

$T=$ absolute temperature of calorimeter and contents

$V=$ volume of the calorimeter 


\section{Journal of Research of the National Bureau of Standards}

$v=$ volume per unit mass

$W=$ molecular weight of the material

$x=$ fraction of the material in the vapor phase, sometimes called dryness.

\section{SUBSCRIPTS}

$a=$ pertaining to the form stable below a transition

$\mathrm{b}=$ pertaining to the form stable above a transition

$\mathrm{c}=$ condensed phase, solid or liquid

$\mathrm{f}=$ pertaining to fusion

$\mathrm{g}=$ gaseous phase

$i=$ pertaining to the $i$ th element of volume of the filling tube

$\mathrm{t}=$ pertaining to the filling tube

$1=$ corresponding to the gross charge $M_{1}$

$2=$ corresponding to the tare charge $M_{2}$.

\section{REFERENCES}

[1] A. Eucken and E. Schröder, Low temperature caloric measurements on a few fluorides $\left(\mathrm{BF}_{3}, \mathrm{CF}_{4}, \mathrm{SF}_{6}\right)$, Z. physik. Chem. [B] 41, 307-319 (1938).

[2] Nathan S. Osborne and Milton S. Van Dusen, Specific heat of liquid ammonia, Bul. BS 14, 397-432 (1918) S313.

[3] A. Eucken, Hand. Experimentalphysik 8, part 1, p. 291-294 (1929).

[4] Nathan S. Osborne, Calorimetry of saturated fluids, J. Opt. Soc. Am. \& Rev. Sci. Instr. 8, 519-540 (1924).

[5] Nathan S. Osborne, Calorimetry of a fluid, BS J. Research 4, 609-629 (1930) RP168.

[6] Henry A. Babcock, The specific heat of ammonia, Proc. Am. Acad. Arts Sci. 55, 323-409 (1920).

[7] J. H. Awbery and Ezer Griffiths, The specific heat of liquid $\mathrm{CH}_{3} \mathrm{Cl}$, Proc. Phys. Soc. 52, 770-776 (1940).

[8] K. Bennewitz and E. Splittgerber, Investigations in the critical region. I. Specific heat of $\mathrm{CO}_{2}$ near the critical point, Z. physik. Chem. 124, 49-65 (1926).

[9] N. S. Osborne, H. F. Stimson, T. S. Sligh, Jr., and C. S. Cragoe, Specific heat of superheated $\mathrm{NH}_{3}$ vapor, BS Sci. Pap. 20, 65-110 (1925) S501.

[10] Harold J. Hoge and Ferdinand G. Brickwedde, Establishment of a temperature scale for the calibration of thermometers between $14^{\circ}$ and $83^{\circ} \mathrm{K}$, J. Research NBS 22, 351-373 (1939) RP1188.

[11] George Rutledge, A reliable method of obtaining the derivative function from smoothed data of observation, Phys. Rev. 40, 262-8 (1932).

Washington, October 24, 1945. 